\title{
INTERFACE SLIP CAUSED BY AN SH-PULSE
}

\author{
Maria Comninou \\ Department of Applied Mechanics and Engineering Science, University of Michigan, Ann Arbor, MI 48109. \\ U.S.A. \\ and \\ J. Dundurs \\ Department of Civil Engineering, University of Michigan, Ann Arbor, MI 48109, U.S.A.
}

(Received 15 February 1979; in revised form 11 June 1979)

\begin{abstract}
Ahatract-The article discusses the reflection and refraction of a plane horizontally polarized (SH) stress pulse by an interface that can slip when the incident pulse is sufficiently strong to break friction. The mathematical nature of the problem depends on the velocity with which the slip zones propagate along the interface. If this velocity is supersonic with respect to both solids, the problem can be solved in closed form. Otherwise, the formulation leads to singular integral equations. A parabolic stress pulse is used to illustrate the result.
\end{abstract}

\section{INTRODUCTION}

We have recently treated the interaction of plane harmonic and horizontally polarized (SH) waves with a unilateral interface[1,2] between two solids. Such waves lead to a periodic array of slip and stick zones. In the present article we consider the refiection and refraction of a plane SH stress pulse that may result in an irregular array of slip and stick zones propagating along the interface.

Suppose that the incident pulse has the phase velocity $c_{0}$ and that its angle of incidence with respect to the interface normal is $\theta_{0}$. Then the interface disturbance propagates with the velocity

$$
v=c_{0} / \sin \theta_{0}
$$

In the analysis one must distinguish between two cases. When $v$ is greater than the phase velocities in both solids (supersonic case), the pulse retains its shape upon reflection and refraction from a bilateral interface that does not slip. Moreover, when localized slip starts, the shear stresses remain unchanged outside the slip zones, and the solution can be obtained by elementary means. When $v$ falls between the speeds of shear waves in the two solids (transonic case) the interaction is more complicated, and the formulation leads to singular integral equations. In the following sections we consider each case separately.

\section{SUPERSONIC CASE}

We consider two elastic solids that are held together by the applied pressure $p^{\infty}$ and are subjected to the applied shear tractions $q^{\infty}$ as shown in Fig. 1 . The shear modulus is denoted by $\mu, c=(\mu / \rho)^{1 / 2}$ is the phase velocity of an SH-wave and bars are used to refer to the physical constants and field quantities associated with the upper half space. The indices $n=0,1,2$ are to distinguish between the three waves in Fig. 1.

The displacement of the incident wave is taken as

$$
u_{3}^{(0)}=C_{0} F\left(y_{0}\right)
$$

where

$$
y_{0}=k_{0}\left(x \cdot p^{(0)}-c_{0} t\right)
$$




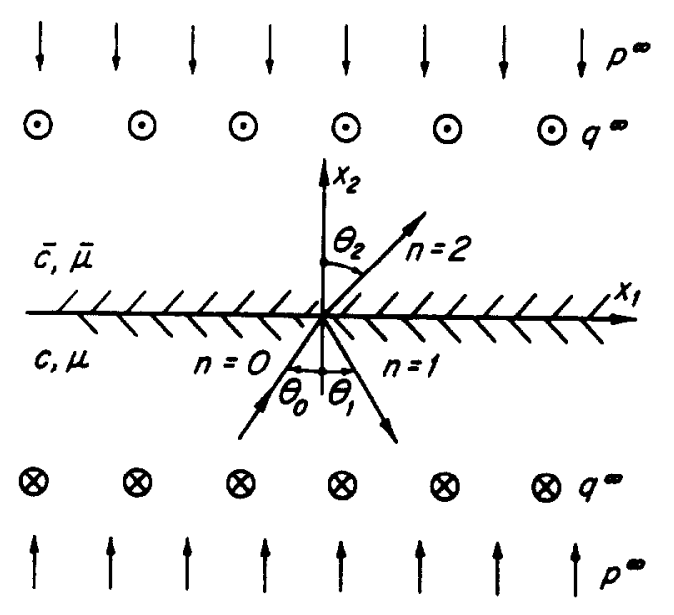

Fig. I. Reflected and refracted SH-pulse.

The unit vector $\mathrm{p}^{(0)}$ defines the direction of propagation of the incident wave [3], and $C_{0}$ is a real constant. The waves refiected and refracted by the bilateral (welded) interface are of the same form as (2), but the vector $p$ and the argument $y$ of the function $F$ must be modified accordingly. The shear traction transmitted by the bilateral interface is determined as in [3]:

$$
\left[\sigma_{23}^{(0)}+\sigma_{23}^{(1)}\right]_{x_{2}=0}+q^{\infty}=\left.\sigma_{23}^{(2)}\right|_{x_{2}=0}+q^{\infty}=\mathscr{A}_{0} f(\eta)+q^{\infty}
$$

where, with $c=c_{0}$,

$$
\eta=k_{0}\left(x_{1} p_{1}^{(0)}-c t\right)
$$

is a coordinate moving along the interface with velocity $v=c / \sin \theta_{0}$, and

$$
f(\eta)=\left.\frac{\mathrm{d} F\left(y_{0}\right)}{\mathrm{d} y_{0}}\right|_{x_{2}=0}
$$

The amplitude $\mathscr{A}_{0}$ of the shear tractions in the bilateral problem is

$$
\mathscr{A}_{0}=\mu C_{0} k_{0} \frac{2 \Gamma \cos \theta_{0} \cos \theta_{2}}{\gamma \cos \theta_{0}+\Gamma \cos \theta_{2}}
$$

with

$$
\Gamma=\bar{\mu} / \mu, \quad \gamma=\bar{c} / c
$$

Moreover, since

$$
\eta_{0}=\eta_{1}=\eta_{2}=\eta
$$

one must have

$$
\begin{gathered}
k_{0} c=k_{1} c=k_{2} \bar{c} \\
\frac{\sin \theta_{0}}{c}=\frac{\sin \theta_{1}}{c}=\frac{\sin \theta_{2}}{\bar{c}} .
\end{gathered}
$$

We take $C_{0}>0$, so that $\mathscr{A}_{0}>0$. We also assume that the two solids adhere before the pulse 
strikes the interface, so that

$$
q^{\infty}<f_{s} p^{\infty}
$$

where $f_{s}$ is the coefficient at static friction.

When the amplitude $\mathscr{A}_{0}$ is sufficiently large, localized slip takes place, and the bilateral solution (4) is no longer applicable and must be corrected. We construct the corrective solution by viewing the slip zones as distributions $B(\eta)$ of screw dislocations moving with velocity $v$ along the interface. The shear traction due to this dislocation distribution is [4]

$$
\tau_{23}=-\frac{\bar{\mu} \zeta_{T} \bar{\zeta}_{T}}{\zeta_{T}+\Gamma \bar{\zeta}_{T}} B(\eta)
$$

where

$$
\zeta_{T}=\left(\frac{v^{2}}{c^{2}}-1\right)^{1 / 2}, \quad \bar{\zeta}_{T}=\left(\frac{v^{2}}{\bar{c}^{2}}-1\right)^{1 / 2}
$$

The slip veocity is related to the dislocation distribution by

$$
V(\eta)=v B(\eta)
$$

The boundary conditions at the interface involve the slip velocity $V(\eta)$ defined by (15), and the total shearing tractions $S(\eta)$ which are nothing else but the sum of (4) and (13). Thus in the stick zones

$$
\begin{gathered}
V(\eta)=0 \\
|S(\eta)|<f_{s} p^{\infty}
\end{gathered}
$$

and in the slip zones

$$
\begin{gathered}
|S(\eta)|=f_{k} p^{\infty} \\
\operatorname{sgn} S(\eta)=\operatorname{sgn} V(\eta)
\end{gathered}
$$

where $f_{k}$ is the coefficient of kinetic friction.

If the slip velocity in the slip zones is positive, (18) can be replaced with

$$
S(\eta)=f_{k} p^{\infty}
$$

or

$$
\mathscr{A}_{0} f(\eta)+q^{\infty}-\frac{\bar{\mu} \zeta_{T} \bar{\zeta}_{T}}{\zeta_{T}+\Gamma_{\bar{\zeta}}} B(\eta)=f_{k} p^{\infty}
$$

The amplitude $\mathscr{A}_{0}$ of the bilateral shear tractions may be expressed as

$$
A_{0}=\frac{2 \bar{\mu} C_{0} k_{0} c \zeta_{T} \bar{\zeta}_{T}}{v\left(\zeta_{T}+\Gamma \bar{\zeta}_{T}\right)}
$$

From (16), (15) and (21) the dislocation distribution is obtained directly as

$$
B(\eta)= \begin{cases}\frac{2 C_{0} k_{0} c}{\mathscr{A}_{0} v}\left[\mathscr{A}_{0} f(\eta)+q^{\infty}-f_{k} p^{\infty}\right], & \text { in slip zones } \\ 0, & \text { in stick zones. }\end{cases}
$$


The shear tractions are

$$
S(\eta)= \begin{cases}f_{k} p^{\alpha}, & \text { in slip zones } \\ \mathscr{A}_{0} f(\eta)+q^{\alpha}, & \text { in stick zones }\end{cases}
$$

and the slip velocity

$$
V(\eta)= \begin{cases}\frac{2 C_{0} k_{0} c}{\mathscr{A}_{0}}\left[\mathscr{A}_{0} f(\eta)+q^{\infty}-f_{k} p^{\infty}\right], & \text { in slip zones } \\ 0, & \text { in stick zones. }\end{cases}
$$

The location and the extent of the slip zones are determined by the inequality (17) and the signum condition (19). The reasoning and the results are quite similar to those for plane harmonic waves[1], and they are shown schematically in Fig. 2 for an incident pulse of a parabolic shape. We note that discontinuities in the slip velocity and shear tractions occur at the leading edges of the slip zones if $f_{s}>f_{k}$. This behavior was also observed in [1] and is characteristic of the supersonic case.

An interesting situation evolves when $f_{k} p^{\infty}<q^{\infty}<f_{s} p^{\infty}$. Once the incident pulse breaks static friction in such case, slip will theoretically not stop after the passage of the pulse.

\section{TRANSONIC CASE}

Assuming that the upper solid is acoustically faster than the lower, the transonic case occurs for angles of incidence in the range

$$
c<\frac{c}{\sin \theta_{0}}<\bar{c}, \text { or } c<v<\bar{c} .
$$

In this range, total reflection is observed, $\mathscr{A}_{0}$ as defined by (7) is complex, and the interface shear traction in the bilateral problem does not retain the shape of the pulse [5]. Since only the effect of localized slip is of interest in this paper, the total reflection of a stress pulse will not be pursued further. Instead the transmitted shear traction is simply taken in the form of (4), but with the understanding that (6) or (7) no longer apply. The dislocation stress is now[4]

$$
\tau_{23}=-\frac{\Gamma \bar{\mu} \zeta T_{T} \psi^{2}}{\zeta_{T}^{2}+\Gamma^{2} \bar{\zeta}^{2}}\left[B(\eta)+\frac{1}{\pi} \frac{\zeta T}{\Gamma \bar{\zeta} \psi} \int_{-\infty}^{\infty} \frac{B(\xi)}{\xi-\eta} \mathrm{d} \xi\right]
$$

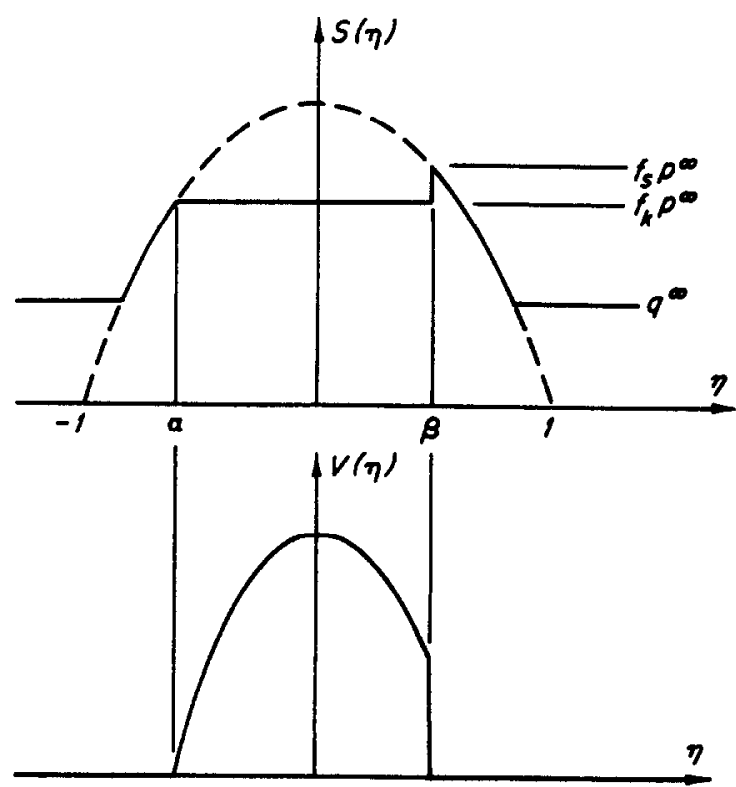

Fig. 2. Shear traction and slip velocity for a parabolic pulse in the supersonic range. 
where

$$
\bar{\zeta} *=\left(1-\frac{v^{2}}{\bar{c}^{2}}\right)^{1 / 2}
$$

and $B(\eta)$ is non-zero only in the slip zones. For the purpose of illustration and without loss of generality we assume that only one slip zone $\alpha<\eta<\beta$ is generated. Applying the boundary conditions as in the previous section, we obtain a Cauchy singular integral equation for $B(\eta)$ :

$$
B(\eta)+\frac{b}{\pi} \int_{a}^{\beta} \frac{B(\eta)}{\xi-\eta} \mathrm{d} \xi=\frac{1}{A_{d}}\left[\mathscr{A}_{0} f(\eta)+q^{\infty}-f_{k} p^{\infty}\right]
$$

where we have set

$$
b=\frac{\zeta T}{\Gamma \bar{\zeta}}
$$

and

$$
A_{d}=\frac{\Gamma \bar{\mu} \zeta \bar{\zeta}^{2} \bar{\psi}^{2}}{\zeta_{T}^{2}+\Gamma^{2} \bar{\zeta}^{2}{ }^{2}}
$$

For the solution of (29) we follow [6]. The inequality precludes stress singularities in the transition from slip to stick, and a bounded solution must be sought. The characteristic function of (29) is then

$$
\begin{aligned}
w(\eta) & =(\beta-\eta)^{\wedge}(\eta-\alpha)^{1-\wedge} \\
\tan \pi A & =-b, \quad\left(\frac{1}{2}<A<1\right) .
\end{aligned}
$$

The solution of the integral equation is

$$
B(\eta)=\frac{1}{1+b^{2}}\left\{\frac{\mathscr{A}_{0}}{d_{d}} f(\eta)-\frac{f_{k} p^{\infty}-q^{\infty}}{\mathscr{A}_{d}}-\frac{b}{\pi} w(\eta) \int_{a}^{\beta} \frac{\frac{\mathscr{A}_{0}}{A_{d}} f(\xi)-\frac{f_{k} p^{\infty}-q^{\infty}}{\mathscr{A}_{d}}}{w(\xi)(\xi-\eta)} \mathrm{d} \xi\right.
$$

provided the consistency condition

$$
\int_{a}^{\beta} \frac{\mathscr{A}_{0} f(\xi)-f_{k} p^{\infty}+q^{\infty}}{w(\xi)} \mathrm{d} \xi=0
$$

is satisfied.

Equation (35) together with the inequality conditions determine the parameters $\alpha$ and $\beta$. To proceed however with the analysis we must specify $f(\eta)$. We may take, for instance, a parabolic shape

$$
f(\eta)=\left\{\begin{array}{cc}
1-\eta^{2} & |\eta|<1 \\
0 & |\eta|>1
\end{array}\right.
$$

It is desirable to obtain a solution in closed form to show clearly the effect of the inequality conditions. Thus we assume that the level of the applied loads and the combination of materials are such that the slip zone falls within the range of the interface tractions in the bilateral problem, or that $|\alpha|<1$ and $|\beta|<1$. The integrations involved are then elementary and can be 
found in [7]. Using (36) in (35) we obtain the following equation for $\alpha$ and $\beta$

$$
-\frac{1}{2}(\beta-\alpha)^{2} A(1-A)+\left(\beta^{2}-\alpha^{2}\right) A+\alpha^{2}=\frac{1}{\mathscr{A}_{0}}\left(\mathscr{A}_{0}-f_{k} p^{\infty}+q^{\infty}\right) .
$$

The dislocation distribution is obtained from (34) as

$$
B(\eta)=\frac{1}{\sqrt{ }\left(1+b^{2}\right)} \frac{A_{0}}{A_{d}} w(\eta)[A(\beta-\alpha)+\alpha+\eta], \quad \alpha<\eta<\beta
$$

The shear traction in the stick zones is

$$
S(\eta)=\mathscr{A}_{0} f(\eta)+q^{\alpha}-\mathscr{A}_{d} \frac{b}{\pi} \int_{\alpha}^{\beta} \frac{B(\xi)}{\xi-\eta} \mathrm{d} \xi, \quad \eta>\beta, \quad \eta<\alpha .
$$

Carrying out the integrations in (39) yields

$$
\begin{aligned}
S(\eta)= & \mathscr{A}_{0} f(\eta)+q^{\infty}-\mathscr{A}_{0}\{[A(\beta-\alpha)+\alpha-\eta][A(\beta-\alpha)+\alpha+\eta] \\
& \left.+[A(\beta-\alpha)+\alpha+\eta]|\eta-\beta|^{A}|\eta-\alpha|^{1-A_{s}} \operatorname{sgn}(\eta-\alpha)+\frac{1}{2}(\beta-\alpha)^{2} A(1-A)\right\}, \quad \eta>\beta, \quad \eta<\alpha .
\end{aligned}
$$

It can be verified from (40) that at $\alpha$ and $\beta$ the shear traction is continuous. Thus the transition from stick to slip will be effected before static friction is reached. We cannot include the effect of static friction without introducing an extra zone along which the transition from static to kinetic friction is achieved according to some continuous friction law. A similar situation was encountered in [2]. It seems that the discontinuous transition required by Coulomb's law can only be accommodated in the supersonic case where the fields exhibit local dependence. In the transonic range, total reflection leads to a smearing out effect which is inconsistent with Coulomb's law of friction for $f_{s} \neq f_{k}$. For the purpose of this paper we will adopt the common assumption of equal coefficients of friction

$$
f_{s}=f_{k}=f
$$

The conditions (17) and (19) must now be enforced. In view of (15), (19) requires that $B(\eta)>0$ or that

$$
A(\beta-\alpha)+2 \alpha>0
$$

To satisfy (17) we must require that the maximum of $S(\eta)$ in the stick zones does not exceed (in absolute value) $f p^{*}$. This is achieved by making the slope of $S(\eta)$ vanish at $\alpha$, yielding

$$
A(\beta-\alpha)+2 a=0
$$

The parameters $\alpha$ and $\beta$ can be determined by solving (37) and (43). From (43) we obtain

$$
\beta=-\frac{2-A}{A} \alpha
$$

and from (44) and (37),

$$
\alpha=-\left[\frac{A^{2}}{6 A-3 A^{2}-2} \frac{\mathscr{A}_{0}+q^{\infty}-f p^{\infty}}{\mathscr{A}_{0}}\right]^{1 / 2}
$$

Note that for slip to start, one must have $A_{0}+q^{\infty}-f p^{\infty}>0$. Also $6 A-3 A^{2}-2<0$ for the given 


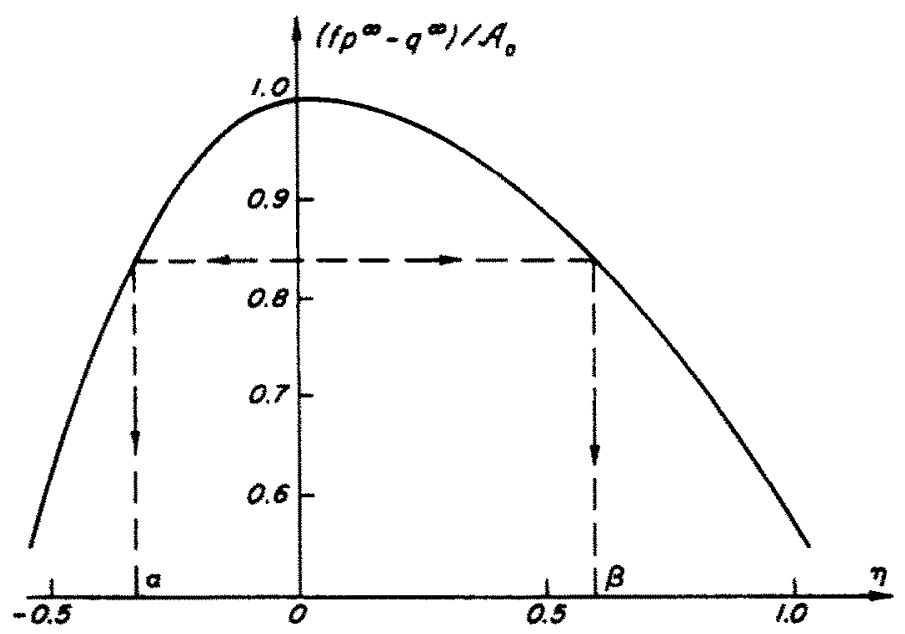

Fig. 3. Variation of $\alpha$ and $\beta$ with $\left(f p^{\infty}-q^{\infty}\right) / A_{0}$ with $A=0.7$ for a parabolic pulse in the transonic case.

range of $A$. In addition, for $\alpha, \beta$ to fall between $(-1,1), A, q^{\infty}$ and $\left(f p \% \mathcal{A}_{0}\right)$ are restricted by

$$
\frac{2-A}{A}\left[\frac{A^{2}}{6 A-3 A^{2}-2}\left(1+\frac{q^{\infty}-f p^{\infty}}{A_{0}}\right)\right]^{1 / 2}<1
$$

Figure 3 shows how $\alpha$ and $\beta$ vary with $\left(f p^{\infty}-q^{\infty}\right) / A_{0}$ for $A=0.7$.

Acknowledgement-The work of one of the authors (MC) was partially supported by the National Science Foundation Grant ENG77-25032.

\section{REFERENCES}

1. E. Chez, J. Dundurs and M. Comninou, Reflection and refraction of SH-waves in presence of slip and friction. Bull. Seism. Soc. Am. 68, 999 (1978).

2. M. Comninou, J. Dundurs and E. Chez, Total reflection of SH-waves in presence of slip and triction. J. Acoust. Soc. Am., 66, 789 (1979).

3. J. Achenbach, Wave Propagation in Elastic Solids. North Holland, Amsterdam (1973).

4. H. M. Berg, J. E. Bloom, H. Ishii, R. H. Marion, D. E. Pease, D. T. Spreng, J. B. VanderSande and J. Weertman, Subsonic, supersonic and transonic dislocations moving on an interface separating two media of differing elastic properties. Fundamental Aspects of Dislocation Theory (Edited by J. A. Simmons, R. deWh and R. Bullough), pp. 71-82. National Bureau of Standards Special Publication 317. Vol. 1 (1970).

5. F. G. Friediander, On the total reflection of plane waves. Quart. J. Mech. Appl. Math. 1, 376 (1948).

6. N. I. Muskhelishvili, Singular Integnal Equations. Noordhof, Leyden (1953).

7. I. S. Gradshteyn and I. M. Ryzhik, Table of Integrals Series and Products. Academic Press, New York (1965). 